\title{
Philosophiques
}

Bernard Baertschi et Kevin Mulligan (dir.), Les nationalismes, Paris, Presses Universitaires de France, collection Éthique et philosophie morale, 2002, 251 pages.

\section{Martin Blanchard}

Volume 31, numéro 1, printemps 2004

Poincaré et la théorie de la connaissance

URI : https://id.erudit.org/iderudit/008947ar

DOI : https://doi.org/10.7202/008947ar

Aller au sommaire du numéro

Éditeur(s)

Société de philosophie du Québec

ISSN

0316-2923 (imprimé)

1492-1391 (numérique)

Découvrir la revue

Citer ce compte rendu

Blanchard, M. (2004). Compte rendu de [Bernard Baertschi et Kevin Mulligan (dir.), Les nationalismes, Paris, Presses Universitaires de France, collection

Éthique et philosophie morale, 2002, 251 pages.] Philosophiques, 31(1), 254-258.

https://doi.org/10.7202/008947ar d'utilisation que vous pouvez consulter en ligne.

https://apropos.erudit.org/fr/usagers/politique-dutilisation/ 
accessible aux débutants. Ses conclusions sont cependant claires: la formulation même de la doctrine de l'eidos doit beaucoup, sinon tout, aux apories de l'illusion et de la perspective, dans la mesure où on ne peut qu'y reconnaître une doctrine de la figure. De belles pages sur l'importance de la doctrine de l'âme auraient mérité un traitement autonome.

Il revenait enfin à Francesco Fronterotta d'exposer la critique aristotélicienne. Dans un livre qui se termine par un texte de Cherniss, dont l'ouvrage central est l'exposé de cette question, le défi était considérable, et cet article le relève de manière lumineuse. L'auteur présente avec précision le corpus des textes d'Aristote où cette critique est formulée, et il en propose une interprétation systématique, en en reprenant de manière formelle les arguments. Cette partie de son article est remarquable de clarté, et le recours au dialogue de jeunesse d'Aristote, De Ideis, tout à fait pertinent. Comme l'auteur le dit avec justesse, la critique aristotélicienne était impitoyable, et elle a conduit Aristote à sa propre conception de l'eidos dans les livres Z-H de la Métaphysique. C'est dans l'exposé de sa conception de la forme qu'on trouve en fait la vraie réponse à Platon.

Ce recueil apporte à la discussion contemporaine un instrument de travail de premier calibre; il est complété de deux courts index, et on peut regretter l'absence de bibliographie (légèrement compensée par des notes souvent très substantielles à cet égard). L'érudition sur ces questions, comme pour tant d'autres, est devenue immaîtrisable, et ce livre fournit un guide sûr pour y faire son chemin.

GEORGES LEROUX

Université du Québec à Montréal

\section{Bernard Baertschi et Kevin Mulligan (dir.), Les nationalismes, Paris, Presses Universitaires de France, collection Éthique et philosophie morale, 2002, 251 pages.}

Cet ouvrage propose quelques pistes pour remédier aux conflits ethniques et nationaux tout en rejetant la solution, jugée inadéquate, qui consiste à éliminer les particularismes de l'espace politique public. Il s'agit d'un recueil rassemblant des textes écrits durant la décennie précédente. L'équilibre recherché est celui d'un "amour du pays» qui ne reconduit pas les tares du "préjugé national ", pour reprendre un mot d'Adam Smith (p. 4).

Le recueil est divisé en trois parties. La première, intitulée Les forces $d u$ nationalisme, contient les textes de Philip Gerans, qui compare diverses formes historiques de nationalisme; de David Miller, qui défend la "nationalité » et de Bernard Baertschi, sur "Le charme secret du patriotisme". La seconde, Les faiblesses $d u$ nationalisme, contient ceux de Daniel M. Weinstock, sur une défense morale du nationalisme; de Ninad Miscevic, sur les difficultés à justifier le nationalisme et d'Elmar Holeinstein sur le concept de Kulturnation. Enfin, la dernière, Quelques remèdes, regroupe les textes de Markus Haller sur le multiculturalisme, de Barry Smith, qui analyse la nation à partir de sa "spatialité » et enfin, de Murray N. Rothbard, sur une conception libertarienne de la nation.

Cette élégante tripartition vise à déployer à la fois les forces et les faiblesses $\mathrm{du}$ nationalisme en proposant, finalement, des solutions qui s'inspirent d'une 
réflexion sur les arguments des sections précédentes. Dans ce qui suit, je m'intéresserai aux contributions les plus prometteuses, soit celles de Miller, Weinstock et Smith. Ce parcours me conduira à conclure que, malgré la qualité de ces textes et de quelques autres, les objectifs que les responsables du recueil se sont donnés n'ont pas été entièrement atteints.

Dans la première section, la contribution de David Miller, «Une défense de la nationalité ", reproduit un article publié en 1993, mais qui demeure toujours d'actualité. Miller y défend l'idée d'une identité nationale — la «nationalité » qui, parce qu'elle constituerait l'attachement politique le plus significatif et le plus contraignant, se distinguerait de toute autre identité collective. Ce type d'attachement engagerait tout citoyen à conduire ses réflexions sur la justice en se rapportant à son identité nationale, bien que cela ne l'empêche pas de reconnaître une valeur intrinsèque possédée par tout être humain. Miller cherche ainsi à trouver un équilibre entre deux tendances qu'il nomme le quotidien (se référant ici à Hume) et le philosophique.

La nationalité est une identité collective singulière qui se réfère à "une communauté constituée par une croyance mutuelle, étendue dans l'histoire, de caractère actif, liée à un territoire particulier, et estimant être séparée des autres communautés par des traits distincts»(p. 39). Ces éléments distinctifs expliquent en partie ce qui rend la nationalité si importante aux yeux de Miller; par exemple, l'autorité d'un groupe national sur un territoire est un aspect essentiel de l'autodétermination politique, au sens où le rapport d'une nation à un territoire particulier fonde le contrôle légitime de ce même territoire par un État. Mais un autre élément important distingue la nationalité. Toujours selon Miller, la nationalité est une identité préphilosophique qui échappe en partie à la raison. L'identité nationale dépendrait ainsi du "sens préréflexif » que tout individu éprouve d'appartenir à un «certain groupe historique» (p. 40). La nationalité serait alors constitutive de l'identité personnelle, donnant un sens au devoir moral des individus, en outre, par l'intermédiaire des obligations mutuelles entre co-nationaux. Cependant, il ne faudrait pas concevoir les identités nationales comme étant exclusives (p. 44). Contre ce préjugé, Miller nous enjoint de prendre acte du monde tel qu'il est, un monde où l'identité nationale cohabite avec une pluralité d'identités. Admettons que cette description soit juste; la version de la nationalité de Miller contient-elle les ressources conceptuelles permettant d'expliquer pourquoi il devrait en être ainsi?

Pour que l'on accorde du crédit à sa thèse, Miller doit nous convaincre que la nationalité constitue le meilleur point de vue pour ordonner une société politique où cohabitent les identités. Miller s'appuie toutefois sur une métaéthique qui introduit une difficulté dans son argumentation, dans la mesure où les identités sont autant d'interprétations qui hiérarchisent les valeurs rattachées à leur appartenance nationale. La difficulté consiste dans le fait que Miller ne dégage pas de critères clairs pour arbitrer les conflits d'interprétations (p. 52). Il est certain que Miller rejettera l'idée qu'un tel critère ne dépend pas d'une culture nationale. Par contre, si les critères de justice, d'impartialité et de cohérence sont tirés d'une culture nationale, ceci risque de poser problème lorsque des intuitions concurrentes tirées d'une même culture revendiqueront leur prépondérance. Sur ce problème, le texte de Miller demeure muet.

Dans la seconde partie (Les faiblesses du nationalisme), Daniel Weinstock critique le texte de Miller tout en se demandant s'il existe une défense morale du 
nationalisme. Weinstock propose une définition du nationalisme qui a l'avantage d'être claire et concise: "une doctrine politique selon laquelle la protection et la promotion des intérêts de la "nation" constituent des fins légitimes pour la politique du gouvernement et peuvent, dans certains cas, justifier une restriction des droits individuels» (p. 92-93). Cette définition illustre bien deux points de friction entre nationalisme et libéralisme: la neutralité politique et le statut des droits individuels. Weinstock prône pour sa part un libéralisme pragmatique qui exige la mise entre parenthèses des identités nationales afin de satisfaire "l'intérêt dominant que tous les groupes ont de réaliser la paix sociale» (p. 98). Une difficulté qui surplombe ce pragmatisme est que la paix sociale pourrait fort bien exiger, dans certains cas, la satisfaction des revendications légitimes d'un groupe national - le conflit pouvant même être attisé par ceux qui refusent toute concession de ce type. $\mathrm{Ne}$ se pourrait-il pas que certaines revendications nationalistes aient une fondation rationnelle, au sens où l'entend le libéralisme pragmatique de Weinstock? Certes, Miller rejette d'emblée cette possibilité, mais justement, il aurait fallu examiner la possibilité d'une conception de la neutralité qui n'est pas anti-nationaliste. Le problème est que Weinstock ne semble accorder aucun crédit à une position neutre qui ne serait pas hostile au nationalisme, comme si le nationalisme empêchait de facto la neutralité axiologique corrigeant «les tendances de nos sympathies naturelles» (p. 101).

Le texte de Weinstock souligne indirectement une impasse majeure dans le débat sur le nationalisme. Il peut paraître raisonnable de s'objecter à une restriction des droits individuels; mais le danger perçu dans cette limitation est ici intimement lié à une conception holiste du nationalisme, qui détermine totalement l'identité individuelle et qui dépasse la portée d'une argumentation rationnelle. Cherchant à éloigner ce spectre, les philosophes libéraux font souvent une distinction catégorique entre, d'une part, ce qu'ils condamnent sous le terme de "nationalisme ethnique» et, d'autre part, un «nationalisme civique» hissé au rang de ce qui serait seul désirable (procédé explicite chez Weinstock et dans l'introduction du recueil). On peut certainement comprendre en quoi un élément identitaire aussi rigide que l'ethnicité et qui, de surcroît, se prétend objectif, est incompatible avec une version libérale de l'identité politique individuelle. Pourtant, en déclassant toute forme de nationalisme qui ne fait pas allégeance à une conception robuste des droits individuels, le risque pour les libéraux est que non seulement les nationalistes, lassés de ce rejet, porteront leurs combats en dehors d'une discussion pacifique, mais aussi que des critiques constructives de la neutralité axiologique soient occultées. Le fait est que la nationalité est un moteur important de l'action politique. C'est pourquoi il importe de considérer l'impact d'une identité nationale non holiste sur la structure des institutions politiques.

Il reste que le texte de Weinstock est une excellente présentation des points de friction qui opposent les nationalistes à certains libéraux. Les trois arguments nationalistes qu'analyse Weinstock - concernant la pression des obligations spéciales sur l'agent moral, le rôle de la solidarité nationale dans les politiques sociales et la capacité du nationalisme à être inclusif — rejoignent une bonne partie des débats en philosophie politique anglo-saxonne. Néanmoins, si les philosophes tardent à emboîter le pas aux études sur le nationalisme, comme le déplore Weinstock dans son introduction, c'est peut-être qu'ils refusent trop rapidement au nationalisme une fondation rationnelle; rien n'indique cependant que cette tâche 
soit une chimère. Weinstock reconnaît explicitement la «nature très limitée » de ses conclusions, centrées sur le nationalisme de Miller. Bien que ce type de nationalisme soit très répandu, il n'en épuise pas le sens.

La dernière section, "Quelques remèdes ", cherche justement à répondre aux exigences nationalistes, du moins à certaines d'entre elles. L'introduction laisse pourtant perplexe, lorsque les auteurs, Baertschi et Mulligan, y affirment que la coexistence des identités culturelles passe par une "éradication des cultures particulières au profit d'une forme de cosmopolitisme pur (plus particulièrement: de culture purement cosmopolitique, sans souci particulier d'autre chose que de la tradition civique)»(p. 165). D'une part, cette affirmation contredit le but de l'ouvrage, qui rejette d'emblée les solutions éliminant les particularismes de l'espace politique public. D'autre part, il eut été plus approprié de dire que les trois auteurs de cette section prônent une dédramatisation des questions identitaires: Markus Haller souhaite leur faire jouer un rôle non pas juridique mais politique; Barry Smith suggère d'autres voies que la territorialité; Murray Rothbard propose pour sa part une perspective "anarcho-capitaliste» qui privatise tout territoire et élimine l'État.

Cette section mérite au moins un commentaire sur le texte de Barry Smith, "La géométrie cognitive de la guerre », qui analyse les manières dont un «objet spatial» est transformé en territoire frontalier. Selon Smith, le destin, peut-on dire, d'un objet spatial donné serait de passer d'un statut "dynamique» (tel un terrain aux frontières fluctuantes qu'occupe une armée) à un statut décrété (un État) ou encore à un statut bona fide, qui consiste dans des barrières frontalières naturelles (par exemple, une île). Il s'agirait ensuite de montrer que toute frontière a une origine qui n'est pas naturelle mais construite: "[L]es objets de type dynamique doivent chaque fois venir en premier, c'est-à-dire qu'ils doivent précéder les objets spatiaux délimités avec soin, qu'ils soient décrétés ou bona fide» (p. 206).

Cette démonstration est ensuite transposée au cas de projets nationaux, ceuxci passant d'un statut dynamique (lorsque l'identité nationale fluctue au gré de son autoreprésentation) à un statut décrété ou bona fide (à travers une autorité reconnue et instituée). Toujours selon Smith, une voie prometteuse permettant de reconnaître un statut institutionnel à des nations «fluctuantes" serait d'assouplir la notion de frontières, pour y inclure des formes dites bizarres (qui correspondent moins à une prétendue géographie naturelle qu'à un décret) des territoires "perforés » (pensons au territoire palestinien) et des territoires enclavés (p. 219). Or, il est difficile d'admettre que la solution pour le cas du Québec soit, comme le préconise Smith, de le "perforer" en octroyant un territoire autonome tant aux francophones qu'aux minorités anglophone et autochtones. Le problème serait alors, d'une part, que ces minorités possèderaient un pouvoir d'influence exagéré sur l'issue de négociations et surtout, d'autre part, que plus rien ne viendrait distinguer les groupes entre eux - pire, ce qui les distinguerait serait alors leur pouvoir de négociation, ce qui propulserait des montées aux enchères.

Cette conclusion illustre par la négative la nécessité d'élargir le concept de nation. Une quantité d'auteurs, pensons par exemple à Kai Nielsen, confèrent à la nation un idéal d'autodétermination politique qui demeure irréductible à une autorité territoriale, un sentiment subjectif ou un consentement individuel. Or la plupart des contributions de ce recueil, à l'exception de Miller, refusent de concéder aux nations un droit à l'autodétermination. Certes, l'immense difficulté consiste à 
élargir le concept de nation sans donner prise aux errements destructeurs qu'il a suscités; mais à trop vouloir en restreindre la portée, le résultat est que le statu quo jalousement maintenu par les grandes puissances est renforcé, ce qui à son tour entraîne une radicalisation inquiétante des petites nations.

Cette réflexion est d'autant plus d'actualité qu'à notre époque une politique de la puissance revient en force. Bien que les textes de ce recueil aient tous été écrits avant 1997, il n'était cependant pas impossible de tenir compte de la situation actuelle. Les quinze dernières années ont montré avec éloquence que les accords négociés, robustes et avantageux aux yeux de tous, constituent la meilleure réponse à l'escalade de conflits ethniques et nationaux. À tout le moins, l'introduction du recueil aurait pu souligner les enjeux de la problématique actuelle, ce qu'elle ne fait pas. Certes, on retrouve dans ce recueil un équilibre très ingénieux entre une tradition philosophique de type analytique et une autre de type continental, ce qui permet d'entrevoir des pistes de solution qui transcendent les frontières disciplinaires. Mais il reste que la plupart des arguments s'adressent tout d'abord à ceux pour qui le nationalisme doit se plier à un nationalisme "civique». Les autres concluront qu'il manque à ces réflexions un concept de nation incluant un aspect civique et politique, mais agrémenté par ailleurs de considérations culturelles, historiques et sociales, pour comprendre ce qui autorise les groupes à déterminer leurs affaires comme bon leur semble.

MARTIN BLANCHARD

Université de Montréal

Roger Pouivet, L'œuvre d'art à l'âge de sa mondialisation. Un essai d'ontologie de l'art de masse, Bruxelles, La Lettre volée, collection Essais, 2003, 113 pages.

Cet essai de Roger Pouivet - qui est en quelque sorte l'application d'une réflexion amorcée dans un ouvrage précédent sur l'ontologie de l'art ${ }^{1}$ - porte sur une question souvent laissée de côté. En effet, la question de l'art de masse n'est pas le parent pauvre de la réflexion en philosophie de l'art: elle y fait plutôt figure d'enfant illégitime. L'objectif de cet ouvrage clair et concis n'est pas tant de discuter de la valeur de l'art de masse sur le plan culturel et artistique, mais plutôt de tenter de cerner ce qui est propre à l'art de masse. Assumant au départ que l'art de masse n'appartient pas à la culture au sens classique du terme, l'auteur cherche à montrer que l'art de masse a substantiellement modifié le statut ontologique de l'œuvre d'art: ce qui en fait l'originalité (par rapport à l'art moderne et contemporain qui ont surtout renouvelé les formes d'art), c'est qu'il substitue au culte de l'original la multiplicité systématique (p. 11). Les deux thèses (soit celle de l'art de masse comme "art de l'ubiquité » et celle qui concerne la valeur de l'art de masse) autour desquelles l'ouvrage est articulé seront brièvement présentées ici, pour mettre ensuite en évidence certaines de leurs implications.

1. Pouivet, Roger. (1999) L'ontologie de l'œuvre d'art : une introduction. J. Chambon, $262 \mathrm{p}$. 